\title{
Uso e disponibilidade de recursos medicinais no município de Ouro Verde de Goiás, GO, Brasil ${ }^{1}$
}

\author{
Cristiane Soares Pereira da Silva ${ }^{2,3}$ e Carolyn Elinore Barnes Proença ${ }^{2}$
}

Recebido em 3/04/2007. Aceito em 5/07/2007

\begin{abstract}
RESUMO - (Uso e disponibilidade de recursos medicinais no município de Ouro Verde de Goiás, GO, Brasil). O objetivo do presente estudo foi realizar um levantamento etnobotânico das plantas medicinais usadas por comunidades rurais e urbanas no município de Ouro Verde de Goiás, situado na mesorregião do mato grosso goiano; eleger espécies nativas do bioma Cerrado potenciais para estudos farmacológicos com base na concordância de uso popular corrigida (CUPc); e avaliar se o conhecimento botânico e o cultivo de espécies medicinais em quintais podem ser afetados por classes de idade, gênero, escolaridade, local de nascimento e procedência rural/urbana do informante pelos testes Kruskal-Wallis $(\mathrm{H})$ e Qui-quadrado $\left(\chi^{2}\right)$. Foram selecionados 84 informantes por meio de amostragens aleatórias, sendo efetuadas entrevistas estruturadas. As fontes disponíveis de recursos medicinais foram: quintais, áreas antrópicas, matas de galeria e remanescentes de florestas estacionais. Foram registradas 98 espécies distribuídas em 45 famílias destacando-se em número as exóticas cultivadas. Nos quintais, foram catalogadas 78 espécies cultivadas, sendo 39,7\% para remédios, e demais associações com a alimentação (39,7\%) e a ornamentação (20,5\%). Vinte espécies são adquiridas pelo extrativismo na vegetação do entorno, sendo todas nativas do bioma Cerrado, com exceção de Senna occidentalis, que é invasora. Duas espécies de florestas estacionais (Forsteronia refracta e Celtis iguanaea) apresentaram a CUPc $>50 \%$, evidenciando consensos de uso popular. Verificou-se que $41 \%$ dos informantes da zona rural recorrem ao extrativismo na vegetação nativa, procura que é consideravelmente maior em relação aos informantes da zona urbana (16,7\%). A quantidade de espécies citadas foi significativamente maior entre os informantes que tinham quintal. O número de espécies citadas e a presença de quintal independem do grau de escolaridade, gênero, local de nascimento, idade e zona de procedência rural ou urbana do informante.
\end{abstract}

Palavras-chave: etnobotânica, plantas medicinais, comunidades locais, quintal, espécies nativas

\begin{abstract}
Use and availability of medicinal resources in Ouro Verde de Goiás, Goiás State, Brazil). The goal of this study was to conduct an ethnobotanical survey of the medicinal plants used by rural and urban communities in the town of Ouro Verde de Goiás, situated in the mato grosso goiano meso-region of the state of Goiás; to pinpoint species native to the Cerrado biome with potential for pharmacological studies based on corrected popular use concordance (CUPc); and to determine if ethnobotanical knowledge of medicinal plants from backyards differed by age, gender, education, place of birth and rural versus urban setting of the informant. Statistical tests applied were Kruskal-Wallis $(\mathrm{H})$ and Qui-square $\left(\chi^{2}\right)$. Eighty-four informants were selected by random sampling and interviews were structured. The available sources of medicinal plants were: backyards, disturbed areas, gallery forests and deciduous dry forests. Ninetyeight species, distributed in 45 botanical families, were found, with cultivated exotics outnumbering native plants. In backyards, 78 species were cultivated, of which $39.7 \%$ were cited exclusively as medicinal, the remaining also being reported as food (39.7\%) or ornamentals $(20.5 \%)$. Twenty species were gathered from the surrounding vegetation, all of which are native to the Cerrado biome, except for Senna occidentalis which is weedy. Two species that occur in deciduous dry forest (Forsteronia refracta and Celtis iguanaea) had high CUPc (> 50\%), showing consensus of popular use. Forty-one percent of rural area informants reported gathering medicinal plants from native vegetation, which is significantly more than those in urban areas (16.7\%). The number of species cited by informants with cultivated backyards was significantly greater than those that did not. The number of medicinal plants cited by informants and the presence of a backyard did not differ significantly among informants from different classes of gender, education, place of birth and rural versus urban dwelling.
\end{abstract}

Key words: ethnobotany, medicinal plants, local communities, backyard, native species

\section{Introdução}

As pessoas tendem a utilizar uma grande diversidade de plantas oriundas de diversos sítios ecológicos naturais ou manejados pelo homem, tais como áreas de vegetação nativa, zonas antropogênicas e totalmente descaracterizadas, e quintais (Albuquerque \& Andrade 2002). A preferência por determinada fonte de recurso pode refletir os aspectos sócio-culturais da comunidade (Begossi et al. 2002;

\footnotetext{
1 Parte da Dissertação de Mestrado da primeira Autora

2 Universidade de Brasília, Instituto Central de Ciências, ICC-Sul, Departamento de Botânica, C. Postal 4457, 70919-970 Brasília, DF, Brasil

3 Autor para correspondência: criskrol@terra.com.br
} 
Estomba et al. 2006), bem como as modificações antrópicas no ambiente (Voeks 1996; Amorozo 2002; Gazzaneo et al. 2005). A obtenção de plantas medicinais em áreas de vegetação nativa também pode ser afetada pela disponibilidade temporal dos recursos ou sazonalidade (Albuquerque et al. 2005; Albuquerque 2006) e por fatores relacionados com técnicas tradicionais de reconhecimento e extrativismo (Lozada et al. 2006), podendo criar restrições de uso às espécies nativas.

É nesse contexto que os quintais se destacam por compatibilizarem o acesso e a coleta, constituindo sistemas adaptados às necessidades locais. O quintal é compreendido como um sistema de produção complementar a outras formas de uso da terra e se destaca pelo valor econômico que desempenha na residência, constituindo uma fonte disponível de recursos alimentícios e medicinais (Pasa et al. 2005). Deve-se enfatizar, também, a sua contribuição na conservação da estrutura física e da fertilidade do solo, pois utiliza insumos naturais, como dejetos de animais, restos vegetais, cinzas e terra transportada das matas, a fim de promover o fortalecimento do espaço que é ocupado por uma variedade de plantas (Lok \& Mendez 1998).

Apesar de diversos trabalhos etnobotânicos terem retratado o uso de plantas medicinais, tanto nativas como cultivadas, em diferentes regiões brasileiras, como na Amazônia (Amorozo \& Gély 1988), na Floresta Atlântica (Medeiros et al. 2004; Gazzaneo et al. 2005; Silva \& Andrade 2005) e em comunidades rurais e urbanas do interior do Brasil (Jacoby et al. 2002; Marodin \& Baptista 2002; Arnous et al. 2005; Pasa et al. 2005), pouco foi investigado a este respeito em áreas florestais do bioma Cerrado.

O conhecimento botânico não constitui uma unidade homogênea entre os sexos, sendo, aparentemente, determinado pelo papel social que homens e mulheres desempenham ou pelas experiências pessoais adquiridas no cotidiano (Phillips \& Gentry 1993a). Alguns estudos têm mostrado que as mulheres tendem a conhecer uma riqueza maior de plantas em determinados grupos étnicos (Begossi et al. 2002). Porém, outras pesquisas mostram que o número de espécies conhecidas independe do gênero e da idade (Botrel et al. 2006; Lozada et al. 2006). Informações desse tipo são fundamentais para evidenciar como o conhecimento de plantas se distribui na comunidade e se este pode ser influenciado por aspectos sócioculturais do informante.

Os processos de decisão do ser humano são extremamente complexos e fatores sociais, culturais, ecológicos e econômicos podem afetar, consideravelmente, na escolha e no conhecimento de espécies medicinais. Assim, este estudo teve como objetivo geral contribuir para a elucidação das fontes disponíveis de recursos medicinais no município de Ouro Verde de Goiás, Goiás, Brasil e como objetivos específicos: 1) registrar as espécies cultivadas e as que são obtidas pelo extrativismo em áreas de vegetação nativa; 2) avaliar a concordância de uso popular e eleger espécies medicinais nativas de formações florestais do Cerrado com potencial para estudos farmacológicos; 3 ) verificar se a idade, o grau de escolaridade, o local de nascimento, o gênero e a procedência rural/urbana afetam o conhecimento botânico; e 4) testar se essas variáveis influenciam, significativamente, no cultivo de plantas medicinais em quintais.

\section{Material e métodos}

Área de estudo - O estudo foi realizado na zona urbana e rural do município de Ouro Verde de Goiás, situado na mesorregião do mato grosso goiano e no alto da bacia hidrográfica do Ribeirão João Leite (região de APA conforme Decreto Estadual n. 5.704 de 27/12/2002), estando localizado a $70 \mathrm{~km}$ ao norte da capital do estado, Goiânia. Sua área municipal é de $210 \mathrm{~km}^{2}$ e a população consiste em 4.358 habitantes, distribuídos em 2.565 na zona urbana e 1.793 na zona rural. O município delimita-se pelas coordenadas geográficas $16^{\circ} 09^{\prime}-16^{\circ} 20^{\prime} \mathrm{S}$ e $49^{\circ} 18^{\prime}-49^{\circ} 08^{\prime} \mathrm{W}$. A altitude média encontra-se entre 938 e $1.140 \mathrm{~m}$. Importantes cursos d'água nascem na região, como o Ribeirão João Leite, que contribui com 55\% do abastecimento público de Goiânia (SEMARH/GO 2003).

Embora a vegetação característica do estado de Goiás seja o cerrado sensu stricto, com a predominância de pequenas árvores de troncos retorcidos em meio a um diversificado estrato de ervas, subarbustos e arbustos, a vegetação do município de Ouro Verde de Goiás é caracterizada, tipicamente, por formações florestais, sendo as florestas estacionais semideciduais e as matas de galeria as fitofisionomias mais freqüentes.

A economia local está baseada na agricultura, destacando-se a produção de beterraba, cenoura, vagem, repolho, banana, tomate, milho, arroz e feijão, e a criação de gado de leite e de corte. Estas atividades já ocasionaram a destruição de boa parte da vegetação nativa do município. As principais fontes de emprego na cidade são oferecidas por duas confecções de roupas, três indústrias de cerâmica e pelo comércio 
varejista. Na zona rural as pessoas trabalham, principalmente, nas atividades agropecuárias com a prestação de serviços temporários.

Amostragem dos informantes e metodologia - O trabalho de campo foi realizado entre janeiro e março/2006. O primeiro autor fixou residência na área de estudo, pois o objeto de investigação também se estendeu para os especialistas locais da comunidade, que serão retratados em outro trabalho. Os informantes foram selecionados por técnicas específicas de amostragem aleatória propostas por Albuquerque \& Lucena (2004). Para a seleção das residências na zona rural foi empregada a amostragem por área. Sete regiões próximas a cursos de água, serras ou fazendas tradicionais foram selecionadas. Posteriormente, através de uma amostragem aleatória simples, foram escolhidas de três a dez residências, dependendo do número total de propriedades rurais em cada região. Para a seleção das unidades amostrais na zona urbana, foi empregada a amostragem por conglomerados. Um mapa da sede municipal com a numeração de todas as quadras foi obtido na Prefeitura de Ouro Verde de Goiás, de onde foram sorteadas dez quadras. Posteriormente, um desenho esquemático de cada quadra foi cedido pela Prefeitura e um novo sorteio foi realizado, selecionando-se de duas a quatro residências através de uma amostragem aleatória simples.

Foram entrevistadas 84 pessoas, 42 na zona rural e 42 na zona urbana. A faixa etária esteve entre 20 e 85 anos de idade. $\mathrm{O}$ grau de escolaridade foi baixo: $47,6 \%$ cursaram até a $4^{\mathrm{a}}$ série; $20 \%$ completaram o ensino fundamental; $21,4 \%$ não tinham escolaridade nenhuma; $7 \%$ terminaram o ensino médio; e apenas $4 \%$ tinham nível superior. Os informantes nascidos em Ouro Verde de Goiás somaram 51,2\% da amostra; $28,57 \%$ eram provenientes de outros municípios de Goiás; e 20,23\% de outros estados brasileiros, sendo estes, majoritariamente, de Minas Gerais. Foi garantido o anonimato dos informantes, que atestaram por meio de assinaturas o consentimento em participar da pesquisa. O esforço amostral e a riqueza de espécies citadas foram testados por uma curva de acumulação (SPSS versão 8.0). Apesar do número de informantes ter sido relativamente pequeno, a curva indicou uma assíntota (modelo logaritmo, $\mathrm{p}>0,0001$ ), sugerindo que se aumentasse o número amostral de informantes, nenhum aumento significativo na riqueza de plantas seria observado.

A coleta dos dados se deu por meio de entrevistas baseadas em um formulário estruturado para a obtenção de dados pessoais (idade, local de nascimento, grau de escolaridade, gênero, procedência rural/ urbana) e de informações sobre o aspecto botânico das plantas citadas (nome popular, parte usada, indicação de uso). Informações adicionais foram computadas, como o número de espécies citadas e a presença ou ausência de quintal. Este trabalho entende por presença de quintal a qualquer espaço físico da residência destinado ao cultivo de espécies medicinais.

O material botânico foi coletado e desidratado em estufa, sendo posteriormente encaminhado ao Herbário da Universidade de Brasília (UB) e identificado mediante comparações com exsicatas, envio aos especialistas e consultas bibliográficas. Todas as espécies citadas foram coletadas para a identificação, porém, só foram incorporados no UB os espécimes em bom estado de conservação ou com ramos reprodutivos. O sistema de classificação botânica utilizado foi o Angiosperm Phylogeny Group (APG II) apud Souza \& Lorenzi (2005).

Análise dos dados - Foi verificada a freqüência da origem biogeográfica das plantas medicinais amostradas, sendo consideradas três categorias: exótica, para as espécies introduzidas de outros continentes; nativa, para as espécies do Cerrado citadas na listagem da flora vascular do bioma por Mendonça et al. (1998); e americana, para as espécies do continente americano não citadas nessa listagem. A determinação da origem foi obtida por consultas às obras de Mendonça et al. (1998), Lorenzi \& Matos (2002), Souza \& Lorenzi (2005) e ao site oficial do Missouri Botanical Garden (TROPICOS).

Foi calculada a porcentagem relativa do hábito de vida das espécies coletadas. Determinou-se a porcentagem das espécies cultivadas que também são empregadas para outras finalidades além do uso medicinal, como na alimentação (frutas, condimentos) e na ornamentação. As espécies foram computadas para avaliar o índice de similaridade entre os quintais da área rural e urbana aplicando-se o Quociente de Similaridade de Sørensen (Sørensen 1978).

A concordância de uso popular foi calculada para as plantas medicinais citadas por três ou mais informantes por meio das seguintes etapas, adaptadas de Amorozo \& Gély (1988):

a. Concordância de uso popular (CUP):

$$
\mathrm{CUP}=\mathrm{Fid} / \mathrm{Fe} \times 100
$$

onde, Fid é a frequiência de indicação de uma doença específica para a espécie, e $F e$ é a freqüência de citação da espécie correspondente. 


\section{b. Fator de Correção (FC): \\ $\mathrm{FC}=\mathrm{Ni} / \mathrm{Nsp}$}

onde, $N i$ é o número de informantes que citaram a espécie, e $N s p$ é o número de informantes que mencionaram a espécie mais citada entre as obtidas pelo extrativismo e as que são cultivadas em quintais. Julgou-se necessário calcular separadamente o fator de correção para as espécies obtidas pelo extrativismo e para as espécies cultivadas em quintais residenciais a fim de neutralizar as distorções devido ao número de informantes, pois se verificou que a citação de espécies nativas adquiridas pelo extrativismo esteve condicionada, basicamente, aos informantes da zona rural.

c. Concordância de uso popular corrigida (CUPc): CUPc $=$ CUP $\times$ FC

As espécies com CUPc maior que 50\% apresentam um apreciável consenso de uso popular, o que pode indicar potencial medicinal, funcionando como uma pré-triagem de espécies para estudos etnofarmacológicos. Estudos etnofarmacológicos são aqueles que combinam informações empíricas adquiridas junto às comunidades locais com estudos químicos e farmacológicos realizados por uma equipe especializada (Elisabetsky \& Souza 2004).

A diferença estatística existente entre o número absoluto de espécies citadas e as classes de: a) faixa etária $(20 \neg 36,37 \neg 53,54 \neg 70$ e $71 \neg 87$ anos); b) local de nascimento (Ouro Verde de Goiás, outro município de Goiás, demais estados); c) escolaridade (analfabetos, até $4^{\mathrm{a}}$ série, ensino fundamental, ensino médio/superior); d) gênero (masculino, feminino); e e) zona de procedência (rural, urbana) foi determinada pela aplicação do teste de Kruskal-Wallis (H). Também foi verificado se essas variáveis interferem na presença de quintais através do teste de Qui-quadrado $\left(\chi^{2}\right)$. Adicionalmente, foi verificado se a presença de quintais afeta positivamente o número de espécies citadas pelo teste de Mann-Whitney (Z). Todas as análises foram conduzidas usando o pacote estatístico SPSS (versão 8.0) com intervalo de confiança a $95 \%$.

\section{Resultados e discussão}

Espécies medicinais citadas - Foram registradas 98 espécies medicinais distribuídas em 45 famílias (Tab. 1). As famílias mais representativas foram Asteraceae (13 spp.) e Lamiaceae (11 spp.), resultado que está em concordância com trabalhos etnobotânicos realizados em diferentes regiões do país (Marodin \& Baptista 2002; Gazzaneo et al. 2005; Pasa et al. 2005).
Quanto à origem biogeográfica das espécies, 46\% são exóticas, $32 \%$ são americanas e $21 \%$ são nativas do bioma Cerrado. Foram constatadas diferenças estatísticas significativas quanto à origem das espécies citadas $\left(\chi^{2}=8,837 ; p=0,012\right)$, pois se destacaram em número as espécies introduzidas de outros continentes, sendo possível evidenciar a inegável influência das espécies exóticas na farmacopéia brasileira.

O hábito de vida mais freqüente foi o herbáceo (48\%), seguido do arbóreo (28,6\%), arbustivo (10,2\%), trepadeira $(7,1 \%)$, subarbustivo $(4,1 \%)$ e liana $(2 \%)$. Evidências atuais corroboram a ampla utilização de espécies herbáceas nos sistemas de cura populares, pois as ervas tendem a investir em compostos secundários de alta atividade biológica, como alcalóides, glicosídeos e terpenóides, ao invés de desenvolver sistemas de defesas estruturais e de alto peso molecular, como taninos e ligninas (Stepp \& Moerman 2001).

O maior número de espécies (16 spp.) foi indicado para o tratamento de gripes, podendo inferir que o extenso repertório de plantas empregadas para solucionar tal enfermidade parece ser uma resposta aos longos períodos de estiagem, à baixa umidade relativa do ar e às queimadas no Cerrado, os quais favorecem o aparecimento de doenças relativas ao aparelho respiratório. Além das doenças do aparelho respiratório, também se destacam aquelas relacionadas ao aparelho digestivo, como o observado em Pernambuco (Gazzaneo et al. 2005), no Pará (Amorozo \& Gély 1988) e no Rio de Janeiro (Medeiros et al. 2004).

Através da concordância de uso popular corrigida (CUPc) são apontados os usos mais difundidos e aceitos para uma espécie, pois, provavelmente, são utilizados e reconhecidos por muitas pessoas, o que evidencia maior segurança quanto à sua validade (Vendruscolo $\&$ Mentz 2006). Assim, as espécies com CUPc acima de $50 \%$ podem evidenciar um apreciável potencial de uso medicinal; nesse sentido, as espécies cultivadas em quintais, como Plectranthus amboinicus e Mentha pulegium para gripe, Phyllanthus tenellus para rins e Vernonia condensata para estômago, bem como as plantas adquiridas pelo extrativismo na vegetação do entorno, como Forsteronia refracta para câncer, Celtis iguanaea para infecções urinárias e Senna occidentalis para gripe, apresentam um alto consenso de informações e, conseqüentemente, implicam em maior eficácia quanto ao uso (Tab. 3). Quando uma indicação específica apresenta coerência com outras comunidades, também é possível evidenciar a existência de eficácia medicamentosa para a espécie. 
Tabela 1. Plantas medicinais citadas no município de Ouro Verde de Goiás, GO, Brasil, com os respectivos nomes populares, hábito de vida, origem biogeográfica, zona de procedência, frequiência de citação da espécie (Fe), associações da categoria medicinal e registros de Herbário (UB). Hábito: $\mathrm{H}=$ herbáceo, $\mathrm{Ar}=$ arbóreo, $\mathrm{Ab}=$ arbustivo, $\mathrm{Tr}=$ trepadeira, $\mathrm{Li}=$ liana. Origem biogeográfica: $\mathrm{A}=$ americana, $\mathrm{N}=$ nativa, $\mathrm{E}=$ exótica. Assoc. $=$ Associações da categoria medicinal: $\mathrm{M}=$ medicinal apenas, $\mathrm{M} / \mathrm{O}=$ medicinal e ornamental, $\mathrm{M} / \mathrm{A}=\mathrm{medicinal}$ e alimentar, $\mathrm{M} / \mathrm{M}=$ medicinal e madeireiro. Zona = Zona de procedência da citação da espécie: $\mathrm{U}=$ zona urbana, $\mathrm{R}=$ zona rural, $\mathrm{U} / \mathrm{R}=$ urbana e rural.

\begin{tabular}{|c|c|c|c|c|c|c|c|}
\hline Família/Nome científico & Nome popular & Hábito & Origem & Zona & $\mathrm{Fe}$ & Assoc. & UB \\
\hline \multicolumn{8}{|l|}{ ACANTHACEAE } \\
\hline $\begin{array}{l}\text { Justicia pectoralis var. stenophylla Leon. } \\
\text { ADOXACEAE }\end{array}$ & anador & $\mathrm{H}$ & A & $\mathrm{U}$ & 2 & M & - \\
\hline Sambucus australis Cham. \& Schltdl. & sabugueiro & $\mathrm{Ar}$ & $\mathrm{E}$ & $\mathrm{U} / \mathrm{R}$ & 14 & $\mathrm{M} / \mathrm{O}$ & 20191 \\
\hline ALISMATACEAE & & & & & & & \\
\hline $\begin{array}{l}\text { Echinodorus grandiflorus (Cham. \& Schltdl.) Mich. } \\
\text { AMARANTHACEAE }\end{array}$ & chapéu-de-couro & $\mathrm{H}$ & $\mathrm{N}$ & $\mathrm{U} / \mathrm{R}$ & 3 & M & - \\
\hline Alternanthera dentata (Moench) Stuchlik. Ex R. E. Fr. & terramicina & $\mathrm{H}$ & A & $\mathrm{U} / \mathrm{R}$ & 5 & $\mathrm{M} / \mathrm{O}$ & 20266 \\
\hline Alternanthera sp. & corta-febre & $\mathrm{H}$ & A & $\mathrm{U}$ & 2 & M & 20321 \\
\hline Chenopodium ambrosioides $\mathrm{L}$. & mastruz & $\mathrm{H}$ & A & $\mathrm{U} / \mathrm{R}$ & 23 & M & 20198 \\
\hline Gomphrena globosa $\mathrm{L}$. & perpétua-roxa & $\mathrm{H}$ & $\mathrm{E}$ & $\mathrm{U}$ & 1 & $\mathrm{M} / \mathrm{O}$ & 20292 \\
\hline \multicolumn{8}{|l|}{ ANACARDIACEAE } \\
\hline Myracrodruon urundeuva Allemão & aroeira & Ar & $\mathrm{N}$ & $\mathrm{R}$ & 1 & $\mathrm{M} / \mathrm{M}$ & - \\
\hline Spondias mombin L. & sirigüela & $\mathrm{Ar}$ & A & $\mathrm{U}$ & 1 & $\mathrm{M} / \mathrm{A}$ & - \\
\hline \multicolumn{8}{|l|}{ APIACEAE } \\
\hline Foeniculum vulgare Mill. & erva-doce & $\mathrm{H}$ & $\mathrm{E}$ & $\mathrm{U} / \mathrm{R}$ & 10 & $\mathrm{M} / \mathrm{A}$ & 20194 \\
\hline Petroselinum crispum (Mill.) Nyman ex A.W. Hill. & salsa & $\mathrm{H}$ & $\mathrm{E}$ & $\mathrm{U}$ & 3 & $\mathrm{M} / \mathrm{A}$ & 20227 \\
\hline \multicolumn{8}{|l|}{ APOCYNACEAE } \\
\hline Vinca rosea L. & boa-noite & $\mathrm{H}$ & $\mathrm{E}$ & $\mathrm{U}$ & 1 & $\mathrm{M} / \mathrm{O}$ & 20267 \\
\hline Forsteronia refracta Müll. Arg. & cipó-leiteiro & $\mathrm{Li}$ & $\mathrm{N}$ & $\mathrm{R}$ & 10 & M & 20317 \\
\hline \multicolumn{8}{|l|}{ ARACEAE } \\
\hline $\begin{array}{l}\text { Philodendron bipinnatifidum Schott ex Endl. } \\
\text { ARISTOLOCHIACEAE }\end{array}$ & cipó-imbé & $\mathrm{Sb}$ & $\mathrm{N}$ & $\mathrm{R}$ & 2 & $\mathrm{M} / \mathrm{O}$ & - \\
\hline Aristolochia sp. & cipó-maravilha & $\mathrm{Li}$ & $\mathrm{N}$ & $\mathrm{R}$ & 1 & M & 20233 \\
\hline \multicolumn{8}{|l|}{ ASPHODELACEAE } \\
\hline \multicolumn{8}{|l|}{ ASTERACEAE } \\
\hline Acanthospermum australe (Loefl.) Kuntze & carrapicho & $\mathrm{H}$ & $\mathrm{N}$ & $\mathrm{U}$ & 1 & M & 20222 \\
\hline Achillea millefolium $\mathrm{L}$. & sabugueirinho & $\mathrm{H}$ & $\mathrm{E}$ & $\mathrm{U} / \mathrm{R}$ & 4 & M & 20278 \\
\hline Ageratum conyzoides $\mathrm{L}$. & menstrasto & $\mathrm{H}$ & A & $\mathrm{U} / \mathrm{R}$ & 6 & M & 20189 \\
\hline Artemisia absinthium $\mathrm{L}$. & losma & $\mathrm{H}$ & $\mathrm{E}$ & $\mathrm{U} / \mathrm{R}$ & 4 & M & 20253 \\
\hline A. camphorata Vill. & alcanfor & $\mathrm{H}$ & A & $\mathrm{U} / \mathrm{R}$ & 5 & M & 20201 \\
\hline Baccharis trimera (Less) DC. & carqueja & $\mathrm{H}$ & A & $\mathrm{U} / \mathrm{R}$ & 3 & M & 20261 \\
\hline Bidens pilosa $\mathrm{L}$. & picão & $\mathrm{H}$ & A & $\mathrm{U} / \mathrm{R}$ & 1 & M & 20205 \\
\hline Mikania glomerata Spreng. & guaco & $\operatorname{Tr}$ & A & $\mathrm{U} / \mathrm{R}$ & 6 & M & - \\
\hline Pluchea sagittalis (Lam.) Cabrera & quitoco & $\mathrm{H}$ & A & $\mathrm{U}$ & 2 & M & 20242 \\
\hline Soliva sp. & marcelinha & $\mathrm{H}$ & $\mathrm{E}$ & $\mathrm{U} / \mathrm{R}$ & 7 & M & - \\
\hline Tanacetum parthenium (L.) Sch. Bip. & artemígio & $\mathrm{H}$ & $\mathrm{E}$ & $\mathrm{U} / \mathrm{R}$ & 5 & $\mathrm{M} / \mathrm{O}$ & 20243 \\
\hline Vernonia condensata Baker & boldo & Ar & $\mathrm{E}$ & $\mathrm{U} / \mathrm{R}$ & 16 & M & - \\
\hline$V$. ferruginea Less. & assa-peixe-branco & $\mathrm{Ab}$ & $\mathrm{N}$ & $\mathrm{U} / \mathrm{R}$ & 11 & M & - \\
\hline \multicolumn{8}{|l|}{ BIXACEAE } \\
\hline Bixa orellana $\mathrm{L}$. & urucum & Ar & A & $\mathrm{R}$ & 1 & $\mathrm{M} / \mathrm{O}$ & - \\
\hline CAMPANULACEAE & & & & & & & \\
\hline $\begin{array}{l}\text { Centropogon cornutus (L.) Druce } \\
\text { CANNABACEAE }\end{array}$ & bico-de-beija-flor & $\mathrm{Sb}$ & $\mathrm{N}$ & $\mathrm{R}$ & 1 & $\mathrm{M} / \mathrm{O}$ & 20277 \\
\hline $\begin{array}{l}\text { Celtis iguanaea (Jacq.) Sarg. } \\
\text { CANNACEAE }\end{array}$ & esporão-de-galo & $\mathrm{Ab}$ & $\mathrm{N}$ & $\mathrm{U} / \mathrm{R}$ & 6 & M & 20204 \\
\hline $\begin{array}{l}\text { CANNACEAE } \\
\text { Canna sp. }\end{array}$ & & & & & & & \\
\hline $\begin{array}{l}\text { Canna sp. } \\
\text { CARICACEAE }\end{array}$ & embirí & $\mathrm{H}$ & A & $\mathrm{U} / \mathrm{R}$ & 2 & $\mathrm{M} / \mathrm{O}$ & - \\
\hline $\begin{array}{l}\text { Carica papaya } \mathrm{L} . \\
\text { CARYOCARACEAE }\end{array}$ & mamão & $\mathrm{Ab}$ & A & $\mathrm{U} / \mathrm{R}$ & 5 & $\mathrm{M} / \mathrm{A}$ & - \\
\hline Caryocar brasiliense Cambess. & pequizeiro & Ar & $\mathrm{N}$ & $\mathrm{R}$ & 1 & $\mathrm{M} / \mathrm{A}$ & $\begin{array}{c}- \\
\text { continuc }\end{array}$ \\
\hline
\end{tabular}


Tabela 1 (continuação)

\begin{tabular}{|c|c|c|c|c|c|c|c|}
\hline Família/Nome científico & Nome popular & Hábito & Origem & Zona & $\mathrm{Fe}$ & Assoc. & UB \\
\hline \multicolumn{8}{|l|}{ CONVOLVULACEAE } \\
\hline Operculina macrocarpa (L.) Urb. & amaro-leite & $\operatorname{Tr}$ & A & $\mathrm{U} / \mathrm{R}$ & 3 & M & 20322 \\
\hline \multicolumn{8}{|l|}{ COSTACEAE } \\
\hline Costus spiralis (Jacq.) Roscoe & cana-de-macaco & $\mathrm{H}$ & $\mathrm{N}$ & $\mathrm{R}$ & 2 & $\mathrm{M} / \mathrm{O}$ & 20187 \\
\hline \multicolumn{8}{|l|}{ CRASSULACEAE } \\
\hline Cotylodon sp. & bálsamo, baspim & $\mathrm{H}$ & $\mathrm{E}$ & $\mathrm{U} / \mathrm{R}$ & 13 & $\mathrm{M} / \mathrm{O}$ & - \\
\hline \multicolumn{8}{|l|}{ CUCURBITACEAE } \\
\hline Cucurbita pepo L. & abóbora & $\mathrm{H}$ & $\mathrm{E}$ & $\mathrm{R}$ & 2 & $\mathrm{M} / \mathrm{A}$ & - \\
\hline Momordica charantia L. & são-caetano & $\operatorname{Tr}$ & $\mathrm{E}$ & $\mathrm{U}$ & 2 & M & 20251 \\
\hline Sechium edule (Jacq.) Sw. & chuchu & $\operatorname{Tr}$ & A & $\mathrm{U} / \mathrm{R}$ & 3 & $\mathrm{M} / \mathrm{A}$ & 20228 \\
\hline Sicana odorifera (Vell.) Naud. & croá & $\operatorname{Tr}$ & A & $\mathrm{R}$ & 1 & $\mathrm{M} / \mathrm{A}$ & - \\
\hline \multicolumn{8}{|l|}{ EUPHORBIACEAE } \\
\hline Croton urucurana Baill. & sangra-d'água & Ar & $\mathrm{N}$ & $\mathrm{R}$ & 6 & M & 20234 \\
\hline Phyllanthus tenellus Roxb. & quebra-pedra & $\mathrm{H}$ & A & $\mathrm{U} / \mathrm{R}$ & 13 & M & 20214 \\
\hline Ricinus comunis L. & mamona & Ar & $\mathrm{E}$ & $\mathrm{R}$ & 4 & M & - \\
\hline \multicolumn{8}{|l|}{ FABACEAE/Caesalpinioideae } \\
\hline Hymenaea courbaril L. & jatobá & Ar & $\mathrm{N}$ & $\mathrm{U} / \mathrm{R}$ & 2 & $\mathrm{M} / \mathrm{M}$ & 20226 \\
\hline Senna occidentalis (L.) Link & fedegoso & $\mathrm{Sb}$ & $\mathrm{E}$ & $\mathrm{U} / \mathrm{R}$ & 7 & M & 20220 \\
\hline Tamarindus indica $\mathrm{L}$. & tamarindo & $\mathrm{Ar}$ & $\mathrm{E}$ & $\mathrm{R}$ & 1 & $\mathrm{M} / \mathrm{A}$ & - \\
\hline \multicolumn{8}{|l|}{ FABACEAE/Mimosoideae } \\
\hline Albizia niopoides (Spruce ex Benth.) Burkart & angico-branco & Ar & $\mathrm{N}$ & $\mathrm{R}$ & 1 & $\mathrm{M} / \mathrm{M}$ & - \\
\hline \multicolumn{8}{|l|}{ FABACEAE/Papilionoideae } \\
\hline Desmodium adscendens (Sw.) DC. & amor-seco & $\mathrm{H}$ & $\mathrm{N}$ & $\mathrm{U} / \mathrm{R}$ & 3 & M & 20222 \\
\hline \multicolumn{8}{|l|}{ LAMIACEAE } \\
\hline Aeollanthus suaveolens G. Dong. & catinga-de-mulata & $\mathrm{H}$ & $\mathrm{E}$ & $\mathrm{R}$ & 1 & $\mathrm{M} / \mathrm{O}$ & 20237 \\
\hline Lavandula angustifolia Mill. & alfazema & $\mathrm{H}$ & $\mathrm{E}$ & $\mathrm{U}$ & 1 & M & 20241 \\
\hline Leonurus japonicus Houtt. & mane-turé & $\mathrm{H}$ & $\mathrm{E}$ & $\mathrm{R}$ & 6 & M & 20212 \\
\hline Mentha x piperita L. var. citrata (Ehrh.) Briq. & alevante & $\mathrm{H}$ & $\mathrm{E}$ & $\mathrm{R}$ & 1 & M & 20282 \\
\hline M. pulegium L. & puejo & $\mathrm{H}$ & $\mathrm{E}$ & $\mathrm{U} / \mathrm{R}$ & 14 & M & - \\
\hline M. piperita $\mathrm{L}$. & hortelãzinho & $\mathrm{H}$ & $\mathrm{E}$ & $\mathrm{U} / \mathrm{R}$ & 17 & $\mathrm{M} / \mathrm{A}$ & - \\
\hline Ocimum gratissimum L. & alfavacão & $\mathrm{Ab}$ & $\mathrm{E}$ & $\mathrm{U} / \mathrm{R}$ & 10 & $\mathrm{M} / \mathrm{A}$ & 20211 \\
\hline Plectranthus amboinicus (Lour.) Spreng. & hortelã-gordo & $\mathrm{H}$ & $\mathrm{E}$ & $\mathrm{U} / \mathrm{R}$ & 24 & M & - \\
\hline P. barbatus Andrews & sete-dores & $\mathrm{H}$ & $\mathrm{E}$ & $\mathrm{U} / \mathrm{R}$ & 9 & M & 20265 \\
\hline P. neochilus Schltr. & boldo-legítimo & $\mathrm{H}$ & $\mathrm{E}$ & $\mathrm{R}$ & 1 & M & 20275 \\
\hline Rosmarinus officinalis L. & alecrim & $\mathrm{H}$ & $\mathrm{E}$ & $\mathrm{U} / \mathrm{R}$ & 10 & $\mathrm{M} / \mathrm{A}$ & 20200 \\
\hline \multicolumn{8}{|l|}{ LAURACEAE } \\
\hline Cinnamomum zeylanicum Breyn. & canela & Ar & $\mathrm{E}$ & $\mathrm{U} / \mathrm{R}$ & 4 & $\mathrm{M} / \mathrm{A}$ & 20284 \\
\hline Persea americana Mill. & abacateiro & Ar & A & $\mathrm{U} / \mathrm{R}$ & 6 & $\mathrm{M} / \mathrm{A}$ & - \\
\hline \multicolumn{8}{|l|}{ LECYTHIDACEAE } \\
\hline Cariniana estrellensis (Raddi) Kuntze & bingueiro & Ar & $\mathrm{N}$ & $\mathrm{R}$ & 1 & $\mathrm{M} / \mathrm{M}$ & 20254 \\
\hline \multicolumn{8}{|l|}{ LYTHRACEAE } \\
\hline Punica granatum L. & romã & Ar & $\mathrm{E}$ & $\mathrm{U} / \mathrm{R}$ & 4 & $\mathrm{M} / \mathrm{O}$ & 20271 \\
\hline \multicolumn{8}{|l|}{ MALPIGHIACEAE } \\
\hline Malpighia glabra L. & acerola & Ar & A & $\mathrm{U}$ & 2 & $\mathrm{M} / \mathrm{A}$ & - \\
\hline \multicolumn{8}{|l|}{ MALVACEAE } \\
\hline Gossypium hirsutum L. & algodão & Ar & $\mathrm{E}$ & $\mathrm{U} / \mathrm{R}$ & 8 & $\mathrm{M} / \mathrm{O}$ & 20256 \\
\hline \multicolumn{8}{|l|}{ MORACEAE } \\
\hline Ficus carica $\mathrm{L}$. & figo & Ar & $\mathrm{E}$ & $\mathrm{R}$ & 1 & $\mathrm{M} / \mathrm{A}$ & 20248 \\
\hline Morus nigra L. & amora & Ar & $\mathrm{E}$ & $\mathrm{U} / \mathrm{R}$ & 5 & $\mathrm{M} / \mathrm{A}$ & - \\
\hline \multicolumn{8}{|l|}{ MYOPORACEAE } \\
\hline Capraria biflora L. & chá-da-índia & $\mathrm{H}$ & A & $\mathrm{U}$ & 1 & M & 20238 \\
\hline MYRTACEAE & & & & & & & \\
\hline Eugenia uniflora L & pitanga & Ar & A & $\mathrm{R}$ & 1 & $\mathrm{M} / \mathrm{A}$ & - \\
\hline Psidium guajava L. & goiabeira & $\mathrm{Ar}$ & A & $\mathrm{R}$ & 1 & $\mathrm{M} / \mathrm{A}$ & - \\
\hline Syzygium cumini (L.) Skeels & jamelão & Ar & $\mathrm{E}$ & $\mathrm{U} / \mathrm{R}$ & 2 & $\mathrm{M} / \mathrm{A}$ & - \\
\hline PASSIFLORACEAE & & & & & & & \\
\hline Passiflora alata Curtis & maracujá-doce & $\operatorname{Tr}$ & A & $\mathrm{R}$ & 2 & $\mathrm{M} / \mathrm{A}$ & 20324 \\
\hline
\end{tabular}


Tabela 1 (continuação)

\begin{tabular}{|c|c|c|c|c|c|c|c|}
\hline Família/Nome científico & Nome popular & Hábito & Origem & Zona & $\mathrm{Fe}$ & Assoc. & UB \\
\hline \multicolumn{8}{|l|}{ PLANTAGINACEAE } \\
\hline Plantago major $\mathrm{L}$. & transagem & $\mathrm{H}$ & $\mathrm{E}$ & $\mathrm{U} / \mathrm{R}$ & 11 & M & 20245 \\
\hline \multicolumn{8}{|l|}{ POACEAE } \\
\hline Cymbopogon citratus (DC.) Stapf. & capim-cidreira & $\mathrm{H}$ & $\mathrm{E}$ & $\mathrm{U} / \mathrm{R}$ & 18 & M & - \\
\hline Saccharum officinarum L. & cana-de-açúcar & $\mathrm{H}$ & $\mathrm{E}$ & $\mathrm{R}$ & 2 & $\mathrm{M} / \mathrm{A}$ & - \\
\hline Zea mays L. & milho & $\mathrm{H}$ & A & $\mathrm{R}$ & 1 & $\mathrm{M} / \mathrm{A}$ & - \\
\hline \multicolumn{8}{|l|}{ POLYGONACEAE } \\
\hline Polygonum punctatum Eliott. & erva-de-bicho & $\mathrm{H}$ & $\mathrm{N}$ & $\mathrm{U} / \mathrm{R}$ & 1 & M & 20293 \\
\hline \multicolumn{8}{|l|}{ ROSACEAE } \\
\hline Eriobotrya japonica (Thunb.) Lindl. & ameixa & Ar & $\mathrm{E}$ & $\mathrm{R}$ & 1 & $\mathrm{M} / \mathrm{A}$ & 20274 \\
\hline \multicolumn{8}{|l|}{ RUBIACEAE } \\
\hline Rudgea viburnoides (Cham.) Benth. & congonha & $\mathrm{Ab}$ & $\mathrm{N}$ & $\mathrm{U} / \mathrm{R}$ & 2 & M & 20275 \\
\hline \multicolumn{8}{|l|}{ RUTACEAE } \\
\hline Citrus aurantifolia (Christm.) Swingle & limão-galego & $\mathrm{Ar}$ & $\mathrm{E}$ & $\mathrm{U} / \mathrm{R}$ & 4 & $\mathrm{M} / \mathrm{A}$ & - \\
\hline C. aurantium $\mathrm{L}$. & laranja-da-terra & $\mathrm{Ar}$ & $\mathrm{E}$ & $\mathrm{R}$ & 1 & $\mathrm{M} / \mathrm{A}$ & - \\
\hline C. medica $\mathrm{L}$. & cidra & $\mathrm{Ar}$ & $\mathrm{E}$ & $\mathrm{R}$ & 3 & $\mathrm{M} / \mathrm{A}$ & 20217 \\
\hline C. sinensis (L.) Osbeck & laranjeira & $\mathrm{Ar}$ & $\mathrm{E}$ & $\mathrm{U} / \mathrm{R}$ & 10 & $\mathrm{M} / \mathrm{A}$ & 20255 \\
\hline Ruta graveolens $\mathrm{L}$. & arruda & $\mathrm{H}$ & $\mathrm{E}$ & $\mathrm{U} / \mathrm{R}$ & 3 & $\mathrm{M} / \mathrm{O}$ & 20218 \\
\hline \multicolumn{8}{|l|}{ SAPINDACEAE } \\
\hline Dilodendron bipinnatum Radlk. & maria-pobre & $\mathrm{Ar}$ & $\mathrm{N}$ & $\mathrm{R}$ & 6 & $\mathrm{M} / \mathrm{M}$ & 20188 \\
\hline \multicolumn{8}{|l|}{ SCROPHULARIACEAE } \\
\hline Buddleia brasiliensis Jacq. ex Spreng. & barbaço & $\mathrm{Ab}$ & $\mathrm{N}$ & $\mathrm{U}$ & 1 & $\mathrm{M} / \mathrm{O}$ & 20260 \\
\hline \multicolumn{8}{|l|}{ SOLANACEAE } \\
\hline Solanum americanum Mill. & erva-moura & $\mathrm{H}$ & A & $\mathrm{R}$ & 1 & M & 20296 \\
\hline S. cernuum Vell. & panacéia & $\mathrm{Ab}$ & A & $\mathrm{U}$ & 1 & $\mathrm{M} / \mathrm{O}$ & 20196 \\
\hline S. lycocarpum A. St.-Hil. & lobeira & $\mathrm{Ab}$ & $\mathrm{N}$ & $\mathrm{R}$ & 1 & M & 20270 \\
\hline S. melongena $\mathrm{L}$. & berinjela & $\mathrm{H}$ & A & $\mathrm{R}$ & 1 & $\mathrm{M} / \mathrm{A}$ & - \\
\hline S. paniculatum $\mathrm{L}$. & jurubeba & $\mathrm{Ab}$ & A & $\mathrm{R}$ & 1 & $\mathrm{M} / \mathrm{A}$ & - \\
\hline \multicolumn{8}{|l|}{ VERBENACEAE } \\
\hline Aloysia oblanceolata Moldenke & camomila & $\mathrm{Ab}$ & A & $\mathrm{U}$ & 1 & $\mathrm{M} / \mathrm{O}$ & 20281 \\
\hline Lippia alba (Mill.) N.E. Br. & erva-cidreira & $\mathrm{Sb}$ & A & $\mathrm{U} / \mathrm{R}$ & 6 & M & 20193 \\
\hline \multicolumn{8}{|l|}{ VITACEAE } \\
\hline Cissus verticillata (L.) Nicholson \& C.E. Jarvis & insulina & $\operatorname{Tr}$ & A & $\mathrm{U} / \mathrm{R}$ & 2 & M & 20294 \\
\hline \multicolumn{8}{|l|}{ ZINGIBERACEAE } \\
\hline Curcuma longa $\mathrm{L}$. & açafrão & $\mathrm{H}$ & $\mathrm{E}$ & $\mathrm{R}$ & 2 & $\mathrm{M} / \mathrm{A}$ & - \\
\hline Zingiber officinale Roscoe & gengibre & $\mathrm{H}$ & $\mathrm{E}$ & $\mathrm{U} / \mathrm{R}$ & 4 & $\mathrm{M} / \mathrm{A}$ & 20316 \\
\hline
\end{tabular}

As indicações de uso para Plectranthus amboinicus, Mentha pulegium, Phyllanthus tenellus, Vernonia condensata e Senna occidentalis, por exemplo, são correspondentes em comunidades distintas no estado de Goiás, como em Goiânia (Rizzo et al. 1990), em Pirenópolis e na Cidade de Goiás (Rizzo et al. 1999), e em Itumbiara (Arantes et al. 2003).

Neste estudo, duas espécies medicinais nativas do bioma Cerrado apresentaram a concordância de uso popular corrigida com valores acima de $50 \%$, sendo: Forsteronia refracta $(\mathrm{CUPc}=91 \%)$ e Celtis iguanaea $(\mathrm{CUPc}=54 \%)$, ambas características de formações florestais do bioma (Tab. 3). Estes valores evidenciam que essas duas espécies nativas merecem atenção especial em estudos etnofarmacológicos por apresentarem consensos de uso terapêutico entre os informantes. A diversidade química e taxonômica do Cerrado é uma das maiores do planeta (Gottlieb \& Borin 1994). Deste modo, ao substituir o empirismo por estudos científicos, novos compostos podem ser isolados das plantas utilizadas em sistemas médicos tradicionais. Atualmente, porém, os ambientes naturais vêm sofrendo grandes modificações pelo homem, e estima-se que mais de $80 \%$ da área territorial do Cerrado se encontra em estágio avançado de antropização e fragmentação, o que torna urgente a realização de novas pesquisas etnobotânicas para o Planalto Central brasileiro, especialmente para as formações florestais do estado de Goiás, que ainda foram pouco investigadas quando ao uso popular de sua flora.

Fontes disponíveis de recursos medicinais - As principais formas para a obtenção de plantas medicinais 
no município de Ouro Verde de Goiás foram: o cultivo doméstico e o extrativismo. A aquisição por compra foi esporadicamente mencionada. Dentre as fontes disponíveis de recursos medicinais, destacaram-se os quintais e a vegetação do entorno, como áreas antrópicas, matas de galeria e florestas estacionais.

Quintais - A riqueza dos quintais pesquisados foi de 78 espécies, entre exóticas e americanas. Porém, observou-se que algumas espécies nativas do Cerrado são frequientemente cultivadas nas residências, como a cana-de-macaco (Costus spiralis), o barbaço (Buddleia brasiliensis) e a erva-de-bicho (Polygonum punctatum). Não houve diferenças estatísticas significativas quanto à presença de quintais entre as residências entrevistadas nas zonas urbana e rural $\left(\chi^{2}=0,429 ; \mathrm{p}=0,513\right)$.

O quociente de similaridade de Sørensen entre os quintais da zona urbana e rural foi de $67 \%$, evidenciando a existência de um número considerável de espécies medicinais comuns. Adicionalmente, ao comparar as floras exóticas citadas entre Ouro Verde de Goiás e Goiânia (Rizzo et al. 1990), foi constatado que $61 \%$ das espécies registradas são comuns aos dois municípios goianos, sugerindo que as espécies exóticas introduzidas de outros continentes são amplamente distribuídas, sendo compartilhadas por diferentes comunidades.

Paralelamente ao uso medicinal, as plantas cultivadas nos quintais também foram mencionadas para outras finalidades (Tab. 2). Dentre as plantas medicinais associadas aos usos alimentar/condimentar, o hortelãzinho (Mentha piperita), a laranjeira (Citrus sinensis), o alecrim (Rosmarinus officinalis), a ervadoce (Foeniculum vulgare), o abacateiro (Persea americana) e a amora (Morus nigra) foram as mais citadas. As plantas medicinais consideradas ornamentais, como o sabugueiro (Sambucus australis), o algodão (Gossypium hirsutum) e o artemígio (Tanacetum parthenium), também foram

Tabela 2. Porcentagem das espécies cultivadas nos quintais associadas à categoria medicinal no município de Ouro Verde de Goiás, GO, Brasil.

\begin{tabular}{lcc}
\hline Associações da categoria medicinal & $\mathrm{N}^{\circ}$ de spp. & $(\%)$ \\
\hline Medicinal apenas & 31 & 39,7 \\
Alimentação & 23 & 39,7 \\
$\quad$ Frutas & 8 & \\
$\quad$ Condimentos & 16 & 20,5 \\
Ornamentação & & \\
\hline
\end{tabular}

consideradas importantes por contribuírem na composição visual dos quintais.

Em Ouro Verde de Goiás, entre as 78 espécies que são cultivadas, $47(60 \%)$ apresentam usos adicionais como alimentícias e/ou ornamentais (Tab. 2). Esses resultados são corroborados pelos dados obtidos por Bennett \& Prance (2000) ao compilar as espécies exóticas usadas no norte da América do Sul (Brasil, Colômbia, Equador e Peru), onde verificaram que do total de 216 espécies amostradas, 155 (72\%) também apresentam usos adicionais na alimentação e ornamentação. Segundo esses autores, as espécies foram introduzidas basicamente para atender às necessidades alimentares e, secundariamente, foram empregadas como remédios, uma vez que muitas espécies exóticas não são usadas como terapêuticas em suas áreas de origem. É por isso que muitas plantas medicinais cultivadas em residências também são empregadas na alimentação, como, por exemplo, o limão, a abóbora, o figo e o açafrão.

Vegetação do entorno das residências - Vinte espécies são obtidas pelo extrativismo e empregadas nas práticas da medicina popular, sendo todas nativas do Cerrado, com exceção de Senna occidentalis. Verificou-se que $41 \%$ dos informantes na zona rural recorrem ao extrativismo, contra $16,7 \%$ na zona urbana. Esta diferença foi estatisticamente significativa $\left(\chi^{2}=5,833\right.$; $\mathrm{p}=0,016)$, sugerindo que a vegetação do entorno da residência constitui uma extensão do quintal para as comunidades rurais e uma fonte de acesso facilitado à flora nativa.

A distribuição das espécies em diferentes ambientes de coleta nos leva a pensar a respeito da destruição da vegetação nativa, fato evidenciado pela presença de algumas espécies que são toleradas ou mesmo protegidas em áreas de pastagens ou de cultivos e que não foram eliminadas por alguma razão especial, seja por suas propriedades medicinais ou pelo fornecimento de sombra. Assim, a presença isolada das espécies Albizia niopoides, Hymenaea courbaril, Dilodendron bipinnatum, Myracrodruon urundeuva e Cariniana estrellensis em áreas totalmente descaracterizadas podem sinalizar o avanço do desmatamento em direção à vegetação primária.

As zonas antropogênicas oferecem uma disponibilidade relativamente alta de recursos medicinais, em especial ervas (Voeks 1996). Nesse sentido, as espécies herbáceas Acanthospermum australe e Desmodium adscendens são freqüentemente coletadas nesses ambientes. Solanum 
Tabela 3. Espécies medicinais ordenadas quanto à concordância de uso popular corrigida (CUPc) maior que 50\% no município de Ouro Verde, GO, Brasil.

\begin{tabular}{llll}
\hline Espécies medicinais & Obtenção & Usos principais & Parte usada \\
\hline Forsteronia refracta M. Arg. & Extrativismo & Câncer & Látex (in natura) \\
Plectranthus amboinicus (Lour.) Spreng. & Quintal & Gripe & Folha (xarope) \\
Senna occidentalis (L.) Link & Extrativismo & Gripe & Raiz (decocção) \\
Vernonia condensata Baker & Quintal & Má digestão & Folha (sumo) \\
Mentha pulegium L. & Quintal & Gripe & Folha (xarope) \\
Celtis iguanaea (Jacq.) Sarg. & Extrativismo & Infecção urinária & Folha/raiz (decocção) \\
Phyllanthus tenellus Roxb. & Quintal & Rins & Parte aérea (decocção) \\
\hline
\end{tabular}

lycocarpum e Vernonia ferruginea também contribuem para a grande procura de recursos terapêuticos em áreas antrópicas, uma vez que são espécies amplamente empregadas no tratamento de doenças relacionadas ao aparelho respiratório, como gripes e bronquites, enfermidades comuns no Brasil Central devido à pronunciada estação seca do bioma Cerrado.

Apenas duas espécies nativas e características de cerrado sensu stricto ou cerradão (Caryocar brasiliensis e Rudgea viburnoides) são coletadas nos municípios vizinhos, pois em Ouro Verde de Goiás há o predomínio de formações florestais.

Dentre as espécies coletadas em matas de galeria, destacaram-se Aristolochia sp., Centropogon cornutus, Croton urucurana, Echinodorus grandiflorus, Philodendron bipinnatifidum e Polygonum punctatum. As matas de galeria são componentes característicos da unidade paisagística local, pois vários cursos d'água nascem no município. Nas florestas estacionais são coletadas as espécies Celtis iguanaea e Forsteronia refracta. A conformação fragmentada das áreas florestais no município representa vestígios de uma distribuição contínua e muito mais ampla no passado. É nesse contexto que os remanescentes de vegetação nativa merecem atenção especial em estudos etnobotânicos, pois oferecem recursos que são utilizados pela população local principalmente para o fornecimento de remédios, madeira e lenha.

Conhecimento $\times$ aspectos sociais dos informantes Ao verificar se fatores relacionados com o gênero, o local de nascimento, a escolaridade, a idade e a zona de procedência do informante interferem no número de espécies citadas, foram obtidos os seguintes resultados com o teste estatístico de Kruskal-Wallis (H). Gênero: foi constatado que homens e mulheres não apresentam diferenças estatísticas significativas quanto ao número de espécies citadas $(\mathrm{H}=1,881$; $\mathrm{p}=0,170$ ), embora várias especulações tenham mostrado que o conhecimento de plantas medicinais parece ser mais evidente entre informantes do sexo feminino, devido ao maior percentual de mulheres entrevistadas em trabalhos etnobotânicas (vide Rizzo et al. 1999; Jacoby et al. 2002; Arnous et al. 2005). Nesta pesquisa, a maioria dos entrevistados também foi do sexo feminino, perfazendo $82 \%$ do total amostrado; e sugere-se que a elevada porcentagem de mulheres entrevistadas pode ser explicada por três fatores: a maioria delas realiza trabalhos domésticos e são mais fáceis de serem encontradas na residência; geralmente são as responsáveis pelo preparo dos remédios caseiros; e acredita-se que os homens estão ausentes no domicílio no período matutino e/ou vespertino em que as entrevistas são efetuadas. Local de nascimento: o número de espécies citadas independe do local de nascimento dos informantes $(H=0,116$; $\mathrm{p}=0,944)$. Grau de instrução: foi constatado que o número de plantas citadas independe da escolaridade $(\mathrm{H}=2,428 ; \mathrm{p}=0,657)$. Faixa etária: não houve diferenças estatísticas significativas entre a idade do informante e o número de plantas citadas $(\mathrm{H}=3,613$; $\mathrm{p}=0,306)$ e resultados similares também foram obtidos por Tridente (dados não publicados) em Porangatu, Goiás, e por Lozada et al. (2006) em uma comunidade rural na Patagônia, Argentina, na qual constataram que o conhecimento de plantas não é influenciado significativamente pela faixa etária. Zona de residência: foi constatado que o número de plantas citadas não apresentou diferenças estatísticas significativas pelo fato do informante pertencer à zona rural ou à zona urbana $(H=1,142 ; \mathrm{p}=0,285)$.

Presença de quintais $\times$ aspectos sociais dos informantes - A presença de quintais na residência 
influenciou positivamente no número de plantas citadas pelo teste de Mann-Whitney $(Z=7,150 ; p<0,001)$. Acredita-se que esse resultado seja devido a duas possíveis causas: 1) fatores complexos relacionados com a história de vida do informante, tais como oportunidade, aptidão, interesse e/ou necessidade, podem afetar a propensão ao cultivo de plantas medicinais e, simultaneamente, influenciar o conhecimento de um maior número de espécies; 2) a presença do quintal aumenta o campo de explanação do informante, podendo agir como um auxílio à memória, o que influencia no número de espécies citadas. É válido ressaltar que as duas causas não são mutuamente exclusivas.

Devido à influência positiva dos quintais sobre o número de espécies medicinais citadas, achamos de interesse investigar a relação existente entre a presença de quintal na residência e as demais variáveis categóricas usadas no tópico anterior. Gênero: foi constatado que homens e mulheres não apresentaram diferenças estatísticas significativas quanto à presença de quintal na residência $\left(\chi^{2}=0,027 ; p=0,870\right)$ embora se tenha constatado que, na maioria das vezes, cabe às mulheres o cultivo das espécies no quintal, bem como as atividades de manutenção envolvidas na rega, na poda e na reposição das mudas. Local de nascimento: foi constatado que ouro-verdenses, goianos de outros municípios e informantes oriundos de outros estados não apresentaram diferenças estatisticamente significativas quanto à presença de quintal $\left(\chi^{2}=0,902\right.$; $\mathrm{p}=0,637)$. Porém, observou-se que a maior proporção de informantes que têm o hábito de cultivar plantas medicinais é proveniente de outras regiões brasileiras e, como muitos mudam de cidade constantemente ou moram de aluguel, normalmente costumam cultivar as plantas em latas e vasilhas para, depois, poderem transportá-las. Por sua vez, esse costume pode ter contribuído, ao longo dos anos, para a ampliação do número de espécies medicinais conhecidas e cultivadas na região, visto que muitas pessoas podem ter trazido plantas de outras localidades, assim como observado por Amorozo \& Gély (1988) em comunidades caboclas no estado do Pará. Escolaridade: foi constatado que analfabetos, alfabetizados até a $4^{\mathrm{a}}$ série, com ensino fundamental e com ensino médio/superior completos não apresentaram diferenças estatísticas significativas quanto a presença de quintal $\left(\chi^{2}=0,840 ; p=0,359\right)$. Observou-se, porém, que quanto menor a escolaridade maior a proporção de informantes que possuem plantas medicinais cultivadas em quintais. No grupo dos analfabetos, por exemplo, $72 \%$ disseram ter o hábito de cultivar plantas medicinais. Faixa etária: informantes de diferentes classes de idade não apresentaram diferenças estatísticas significativas quanto a presença de quintais $\left(\chi^{2}=3,186 ; p=0,364\right)$, porém, a maior proporção de informantes que têm o hábito de cultivar espécies medicinais pertence às faixas etárias superiores à 54 anos. Parece existir uma relação entre idade e modo de aquisição de plantas medicinais. No grupo de informantes com idade entre 54 e 87 anos, $71 \%$ disseram obter as plantas medicinais no quintal próprio. Esses resultados evidenciam que pessoas nessa faixa etária tendem a conservar as práticas da medicina popular tradicional, cujo conhecimento relativo ao uso de plantas é, geralmente, expresso no cultivo das espécies na residência. Em contrapartida, no grupo dos jovens $(20 \neg 36$ anos) e adultos (37 $\neg 53$ anos) apenas $31 \%$ e $44 \%$ dos entrevistados, respectivamente, têm o hábito de cultivar as plantas, recorrendo com freqüência a outras fontes de recursos, tal como o quintal de vizinhos e parentes. Zona de residência: Assim como as demais variáveis utilizadas, foi constatado que informantes da zona rural e urbana não apresentaram diferenças estatísticas significativas quanto à presença de quintais $\left(\chi^{2}=0,064\right.$; $\mathrm{p}=0,801$ ).

Foi possível verificar que o conhecimento de plantas e o cultivo de espécies medicinais em quintais independem dos aspectos sócio-culturais do entrevistado, ainda que tenha havido diferenças percentuais na amostra. $\mathrm{Na}$ área de estudo, o uso de plantas medicinais ocorreu em $79 \%$ da amostra e esse número corresponde aos informantes que enfatizaram a medicina popular como a principal alternativa para a busca da saúde. Os fatores que podem influenciar o conhecimento de plantas são: a) o baixo poder aquisitivo da família; b) a presença de curadores populares na região, como curandeiros, parteiras, benzedeiras e raizeiros, que difundem as práticas da medicina popular tradicional; e c) a pouca infra-estrutura em saúde ofertada pelo município. Em Ouro Verde de Goiás, o posto de saúde é a única unidade de assistência médica convencional e nele há escassez de recursos humanos e físicos para o atendimento da população. Os pacientes que requerem cuidados mais intensivos são encaminhados até os municípios de Anápolis, a $30 \mathrm{~km}$ de distância, ou Goiânia, a 70 km. É em situações de tratamento médico deficiente que se desenvolve um sistema alternativo baseado na tradição popular, fazendo com que os indivíduos assumam, cada vez mais, 
o controle da própria saúde ao diagnosticar e tratar as doenças com remédios caseiros provenientes de espécies nativas ou de seus substitutos que são cultivados em quintais.

\section{Agradecimentos}

À comunidade rural e urbana do município de Ouro Verde de Goiás, pela receptividade e disposição em ajudar e contribuir com o outro, mesmo que esse seja um completo desconhecido; aos companheiros anônimos da comunidade, pelo auxílio e apoio durante os trabalhos de campo; ao Dr. Ray Harley, pela identificação de algumas espécies da família Lamiaceae; ao Dr. Gercino Monteiro Filho, pelas análises estatísticas; aos professores Dr. Ulysses P. Albuquerque e Dr. Eraldo M. Costa Neto, pelas valiosas sugestões e contribuições com este trabalho; à Coordenação de Aperfeiçoamento de Pessoal de Nível Superior (CAPES) e à coordenação do curso de Pós-Graduação em Botânica da Universidade de Brasília, pela bolsa e apoio concedidos à primeira autora.

\section{Referências bibliográficas}

Albuquerque, U.P. \& Andrade, L.H.C. 2002. Conhecimento botânico tradicional e conservação em uma área de caatinga no Estado de Pernambuco, Nordeste do Brasil. Acta Botanica Brasilica 16: 273-285.

Albuquerque, U.P. \& Lucena, R.F.P. (orgs.). 2004. Métodos e técnicas na pesquisa etnobotânica. Recife, LivroRápido/ NUPEEA.

Albuquerque, U.P.; Silva, A.C.O. \& Andrade, L.H.C. 2005. Use of plant resources in a seasonal dry forest (northeastern Brazil). Acta Botanica Brasilica 19: 27-38.

Albuquerque, U.P. 2006. Re-examining hypoteses concernig the use and knowledge of medicinal plants: a study in the Caatinga vegetation of NE Brazil. Journal of Ethnobiology and Ethnomedicine 2: 1-10.

Amorozo, M.C.M. \& Gély, A. 1988. Uso de plantas medicinais pelos caboclos do baixo Amazonas, Barcarena, Pará, Brasil. Boletim do Museu Paraense Emílio Goeldi, Botânica 4: 47-131.

Amorozo, M.C.M. 2002. Uso e diversidade de plantas medicinais em Santo Antonio do Leverger, MT, Brasil. Acta Botanica Brasilica 16: 189-203.

Arantes, A.A.; Caldas, E.R. \& Silva, K.G.A. 2003. O uso de plantas medicinais no município de Itumbiara, Goiás, Brasil. Práxis 3: 43-56.

Arnous, A.H.; Santos, A.S. \& Beinner, R.P.C. 2005. Plantas medicinais de uso caseiro: Conhecimento popular e interesse por cultivo comunitário. Espaço para a Saúde 6: $1-6$.
Begossi, A.; Hanazaki, N. \& Tamashiro, J.Y. 2002. Medicinal plants in the Atlantic Forest (Brazil): knowledge, use and conservation. Human Ecology 30: 281-299.

Bennett, B.C. \& Prance, G.T. 2000. Introduced plants in the indigenous pharmacopoeia of Northern South America. Economic Botany 54: 90-102.

Botrel, R.T.; Rodrigues, L.A.; Gomes, L.J.; Carvalho, D.A.; Fontes, M.A.L. 2006. Uso da vegetação nativa pela população local no município de Ingaí, MG, Brasil. Acta Botanica Brasilica 20: 143-156.

Elisabetsky, E. \& Souza, G.C. 2004. Etnofarmacologia como ferramenta na busca de substâncias ativas. Pp. 107-122. In: C.M.O. Simões; E.P. Schenkel; G. Gosmann; J.C.P. Mello; L.A. Mentz \& P.R. Petrovick (orgs.). Farmacognosia: da planta ao medicamento. $5^{\mathrm{a}} \mathrm{ed}$. Ed. da UFRGS/UFSC.

Estomba D.; Ladio A. \& Lozada M. 2006. Medicinal wild plant knowledge and gathering patterns in a Mapuche community from North-western Patagonia. Journal of Ethnopharmacology 103: 109-119.

Gazzaneo, L.R.S.; Lucena, R.F.P. \& Albuquerque, U.P. 2005. Knowledge and use of medicinal plants by local specialists in an region of Atlantic Forest in the state of Pernambuco (Northeastern Brazil). Journal of Ethnobiology and Ethnomedicine 1: 1-11.

Gottlieb, O. R. \& Borin, M.R.M.B. 1994. The diversity of plants. Where is it? Why is it there? What will it become? Anais da Academia Brasileira de Ciências 66: 205-210.

Jacoby, A.; Coltro, E.M.; Sloma, D.C.; Muller, J.; Dias, L.A.; Luft, M. \& Beruski, P. 2002. Plantas medicinais utilizadas pela comunidade rural de Guaramirim, Município de Irati, Paraná. Revista Ciências Exatas e Naturais 4: 79-89.

Lok, R. \& Mendez, E. 1998. El uso del ordenamiento local del espacio para una clasificación de huertos na Nicaragua. Pp.129-149. In: R. Lok (ed.). Huertos tradicionales de América Central: características, beneficios e importancia, desde um enfoque multidisciplinario. Turrialba, Costa Rica, CATIE/EGUILA/IDR/ETC Andes.

Lorenzi, H. \& Matos, F.J.A. 2002. Plantas medicinais no Brasil: nativas e exóticas cultivadas. Nova Odessa, São Paulo, Instituto Plantarum.

Lozada, M.; Ladio, A. \& Weigandt, M. 2006. Cultural transmission of ethnobotanical knowledge in a rural community of northwestern Patagonia, Argentina. Economic Botany 60: 374-385.

Marodin, S.M. \& Baptista, L.R.M. 2002. Plantas medicinais do município de Dom Pedro de Alcântara, Estado do Rio Grande do Sul: espécies, famílias e usos em três grupos de população humana. Revista Brasileira de Plantas Medicinais 5: 1-9.

Medeiros, M.F.T.; Fonseca, V.S. \& Andreata, R.H.P. 2004. Plantas medicinais e seus usos pelos sitiantes da Reserva Rio das Pedras, Mangaratiba, RJ, Brasil. Acta Botanica Brasilica 18: 391-399.

Mendonça, R.C.; Felfili, J.M.; Walter, B.M.T.; Silva-Júnior, M.C.; Rezende, A.V.; Filgueiras, T.S. \& Nogueira, P.E. 1998. Flora vascular do bioma Cerrado. Pp. 307-556. In: S.M. Sano \& S.P. Almeida (orgs.). Cerrado ambiente e flora. Planaltina, EMBRAPA - CPAC, Brasília. 
Pasa, M.C.; Soares, J.N. \& Guarin-Neto, G. 2005. Estudo etnobotânico na comunidade de Conceição-Açu (alto da bacia do rio Aricá Açu, MT, Brasil). Acta Botanica Brasilica 19: 195-207.

Phillips, O. \& Gentry, A.H. 1993a. The useful plants of Tambopata, Peru: I. Statistical hypotheses tests with a new quantitative technique. Economic Botany 47: 15-32.

Rizzo, J.A.; Monteiro, M.S.R. \& Bitencourt, C. 1990. Utilização de plantas medicinais em Goiânia. Pp. 691-714. In: Anais do XXXVI Congresso Brasileiro de Botânica. Curitiba, 1985. Brasília, Sociedade Botânica do Brasil.

Rizzo, J.A.; Campos, I.F.P.; Jaime, M.C.; Munhoz, G. \& Morgado, W.F. 1999. Utilização de plantas medicinais nas cidades de Goiás e Pirenópolis, Estado de Goiás. Revista de Ciências Farmacêuticas 20: 431-447.

SEMARH/GO - Secretaria de Estado do Meio Ambiente e dos Recursos Hídricos do Estado de Goiás. 2003. Área de Proteção Ambiental - Apa João Leite. Goiânia, Goiás.

Silva, A.J.R. \& Andrade, L.H.C. 2005. Etnobotânica nordestina: estudo comparativo da relação entre comunidades e vegetação na Zona do Litoral - Mata do Estado de Pernambuco, Brasil. Acta Botanica Brasilica 19: 45-60.
Sørensen, T. 1978. A method of establishing groups of equal amplitude in plant sociology based on similarity of species contents. In: R. McIntosh (ed.). Phytosociology. Pennsylvania, Dowdew, Hutchinson \& Ross, Inc.

Souza, V.C. \& Lorenzi, H. 2005. Botânica Sistemática. Guia ilustrado para identificação das famílias de Angiospermas da flora brasileira, baseado em APG II. Nova Odessa, São Paulo, Instituto Plantarum.

Stepp, J.R. \& Moerman, D.E. 2001. The importance of weeds in ethnopharmacology. Journal of Ethnopharmacology 75: $19-23$.

TROPICOS - Nomenclatural Data Base. Missouri Botanical Garden. Disponível em: <http://mobot.mobot.org/w3t/ Search/vast.html/> (Acesso em: 25/outubro/2006).

Vendruscolo, G.S. \& Mentz, L.A. 2006. Estudo da concordância das citações de uso e importância das espécies e famílias utilizadas como medicinais pela comunidade do bairro Ponta Grossa, Porto Alegre, RS, Brasil. Acta Botanica Brasilica 20: 367-382.

Voeks, R.A. 1996. Tropical Forest healers and habitat preference. Economic Botany 50: 382-400. 